\section{The shortcut strategy for beta thalassemia prevention}

\author{
Narutchala Suwannakhon, ${ }^{1}$ \\ Khajohnsilp Pongsawatkul, ${ }^{2}$ \\ Teerapat Seeratanachot, ${ }^{3}$ \\ Khwanruedee Mahingsa, ${ }^{4}$ \\ Arunee Pingyod, ${ }^{4}$ \\ Wanwipa Bumrungpakdee, ${ }^{4}$ \\ Torpong Sanguansermsri ${ }^{4}$
}

${ }^{1}$ Discipline of Biology, School of Science,

University of Phayao; ${ }^{2}$ Division of

Obstetrics and Gynecology, Phayao

Hospital; ${ }^{3}$ Discipline of Biochemistry,

School of Medical Science, University of

Phayao; ${ }^{4}$ Thalassemia Unit, University

of Phayao, Thailand

\begin{abstract}
We propose antenatal blood tests using high-resolution DNA melting (HRM) analysis for beta thalassemia mutation detection after hemoglobin $\mathrm{A}_{2}$ estimation as a modified strategy for the identification of beta thalassemia at-risk couples. Antenatal blood samples of 1,115 couples were transferred from the antenatal care clinic. Hemoglobin $\mathrm{A}_{2}$ was quantified, and proportions $\geq 3.5 \%$ were further assessed for beta thalassemia mutation using HRM analysis. Twelve types of beta thalassemia mutations, including hemoglobin E, were identified. There were 23 couples who were detected as at-risk. All at-risk couples were identified within 7 working days after sample receipt. Prenatal diagnosis revealed 6 affected fetuses. One fetus was homozygous CD17 (A$\mathrm{T}$ ), and five fetuses exhibited beta ${ }^{0}-$ thalassemia/hemoglobin E disease. These results were consistent with the outcomes calculated using the Hardy-Weinberg equation. Antenatal blood tests for mutation detection using high-resolution DNA melting analysis after hemoglobin $\mathrm{A}_{2}$ estimation is a feasible laboratory method for the recruitment of couples with a fetus that is at risk for beta thalassemia. This modified strategy is cost-effective and may be beneficial for use in a beta thalassemia prevention program.
\end{abstract}

\section{Introduction}

There is a high prevalence of alpha, beta thalassemia, hemoglobin E, hemoglobin Constant Spring and other abnormal hemoglobin-carrier conditions in Thailand. ${ }^{1}$ The combination of these genes results in tha- lassemia disease with clinical heterogeneity. Beta thalassemia disease is the most common hereditary chronic anemia, and cases are observed nationwide. Homozygous beta ${ }^{0}$-thalassemia and/or compound heterozygous beta ${ }^{0}$-thalassemia results in a live birth and is the full form of the disease. The patient is almost always dependent on blood transfusions and requires daily iron chelation therapy. Beta ${ }^{0}$-thalassemia/hemoglobin E disease is a severe form of chronic anemia with progressive iron overload. Beta ${ }^{0}$-thalassemia/hemoglobin $\mathrm{E}$ disease requires occasional blood transfusions and daily iron chelation therapy. ${ }^{2-7}$ A total of 3000 to 4000 new cases of beta thalassemia disease are diagnosed annually. The annual treatment cost of these patients is enormous, and it is a substantial national economic burden. Therefore, the national thalassemia prevention program was implemented in 2001. Beta thalassemia major and beta ${ }^{0}$-thalassemia/hemoglobin E disease are included in the national thalassemia prevention program. This program is cost-free, and it has been used in all antenatal couples in combination with thalassemia counseling and lab tests for couples who received a prenatal diagnosis that their fetus is at-risk for the disease. $^{8}$

There are approximately 60,000 births annually in the Northern provinces, including 60 cases of beta thalassemia major and 180 cases of beta $^{0}$-thalassemia/hemoglobin E disease. ${ }^{9,10}$ A 10-year surveillance report (2005 to 2015) of Thalassemia Labs Unit, Department of Pediatrics, Faculty of Medicine, Chiang Mai University, Chiang Mai, Thailand revealed that the mean annual detection of beta thalassemia major and beta ${ }^{0}$-thalassemia/hemoglobin $\mathrm{E}$ disease was 27 and 85 cases, respectively (Phusua and Suanta 2017, personal communication). Newly affected births of beta thalassemia major and beta ${ }^{0}$-thalassemia/hemoglobin $\mathrm{E}$ disease from various provincial hospitals were reported. Analysis of clinical records indicated a service failure that was primarily due to an inadequate local screening policy. This study proposes a modified carrier screening strategy via direct assessment of an antenatal blood sample and high-resolution DNA melting analysis after hemoglobin $\mathrm{A}_{2}$ quantification.

\section{Materials and Methods}

\section{Samples}

Antenatal blood samples of 1,115 couples were collected from the antenatal care clinic of the Provincial Hospital, Prayao,
Correspondence: Narutchala Suwannakhon, Discipline of Biology, School of Science, University of Phayao, Phayao, Thailand.

Tel.: +66.0810296551 - Fax: +66.054466724. E-mail: narutchala@gmail.com

Key words: Shortcut strategy, Beta thalassemia prevention, Hemoglobin E, Hemoglobin A2.

Acknowledgments: the authors would express their sincere thanks to Professor Dr. Monton Sanguansermsri, President of the Prayao University for his generous allocation of the University Endowment Fund that supported this project.

Contributions: NS, TS, conception and design; NS, KP, acquisition of data; NS, KM, AP, WB, TS, development of methodology and sample tests; NS, TS, KP, analysis and interpretation of data; NS, TS, writing, review and/or revision of the manuscript; NS, study supervision; TS, research consultants.

Conflict of interest: the authors declare no potential conflict of interest.

Funding: none.

Received for publication: 10 December 2017. Accepted for publication: 15 May 2018

This work is licensed under a Creative Commons Attribution-NonCommercial 4.0 International License (CC BY-NC 4.0).

CCopyright N. Suwannakhon et al., 2018

Licensee PAGEPress, Italy

Hematology Reports 2018; 10:7530

doi:10.4081/hr.2018.7530

Thailand from 2015 to 2016. The average fetal age was 10-12 weeks. Ethical approval was obtained from the Medical Ethics Committee of the Prayao University (3/011/59). Informed consent forms were collected from pregnant women and their spouses prior to blood collection and prenatal diagnosis.

\section{Workflow}

Hemoglobin $\mathrm{A}_{2}$ was quantified in all blood samples using Q-Sepharose (GE Healthcare Bio Sciences AB, Uppsala, Sweden) micro-column chromatography and capillary electrophoresis simultaneously (SEBIA - Parc Technologique Léonard de Vinci CP 8010 Lisses - 91008 Evry Cedex France). Hemoglobin $\mathrm{A}_{2}$ levels $\geq 3.5 \%$ were further assessed for beta thalassemia mutation using high-resolution DNA melting analysis. Couples at-risk for beta thalassemia were identified. The recruitment of couples at-risk was arranged for completion within 7 days after sample receipt. All at- 
risk couples received a prenatal diagnosis via amniocentesis within 16 weeks of gestation. Fetal DNA diagnosis was performed using high-resolution DNA melting analysis and a direct DNA sequencing technique. Babies were monitored for one year after birth.

\section{Q-Sepharose micro-column chro- matography}

Q-Sepharose $0.5 \mathrm{~mL}$ was packed into the tip of a well-designed disposable microcolumn with a $10-\mathrm{mL}$ reservoir. QSepharose micro-column chromatography was equilibrated with $10 \mathrm{~mL}$ of working buffer $(0.05 \mathrm{M}$ Tris-HCl- KCN, $\mathrm{pH} 8.5)$. The hemolysate was prepared by mixing 20 $\mu \mathrm{L}$ of whole blood with $2 \mathrm{~mL}$ of the working buffer. One milliliter of hemolysate was applied to the micro-column. The unbound hemoglobin was washed out using $2 \mathrm{~mL}$ of the working buffer. Hemoglobin $\mathrm{A}_{2}$ was eluted with $4 \mathrm{~mL}$ of eluting buffer $(0.05 \mathrm{M}$ Tris-HCl- KCN and $12 \mathrm{mM} \mathrm{NaCl}, \mathrm{pH}$ 8.5). The eluate was measured at an absorbance of $415 \mathrm{~nm}$ and was designated OD-1. The reference OD-2 was an absorbance of 415 $\mathrm{nm}$, which was created by mixing $1 \mathrm{~mL}$ of the previously prepared hemolysate with 3 $\mathrm{mL}$ of the working buffer. The percentage of hemoglobin $\mathrm{A}_{2}$ (hemoglobin E) was calculated using the following formula: (OD-1 $\div$ OD- 2$) \times 100$. The mean $( \pm$ SD) hemoglobin $\mathrm{A}_{2}$ proportions in the normal and genetically demonstrated beta thalassemia heterozygotes were $2.7 \pm 0.4 \%$ and $6.3 \pm 1.23 \%$, respectively, whereas the proportion of hemoglobin E heterozygotes was $23.25 \pm 4.13 \% .^{11}$

\section{Capillary electrophoresis system}

Hemoglobin separation and quantification of hemoglobin types were performed using the CAPILLARYS 2 FLEX PIERCING equipped with 8 capillaries according to the manufacturer's instructions. Briefly, the blood sample was added to a lysis solution, and an aliquot was introduced using aspiration at the anodic end into the capillary tube prior to application of $9,800 \mathrm{~V}$. Detection of hemoglobin was performed via measurement of the absorbance at $415 \mathrm{~nm}$ at the cathodic end of the capillary tube. The hemoglobin $\mathrm{A}_{2}$ fraction was adjusted at the end of the analysis to appear in zone 9 in the middle of the window. The relative quantity (percent of total hemoglobin) and presumptive identification of the hemoglobin types (located in various zones from zone 1 to 15) were recorded from the resulting electropherogram. The positions of different hemoglobin types were identified in the pertinent zones. The mean $( \pm \mathrm{SD})$ hemoglobin $\mathrm{A}_{2}$ proportions in the normal and genetically proven beta thalassemia heterozygotes were $2.8 \pm 0.3 \%$ and $5.8 \pm 0.7 \%$, respectively, and the proportion of hemoglobin E heterozygotes was $24.0 \pm 3 \%$. $^{12-14}$

\section{DNA isolation}

Genomic DNA was isolated from 200 $\mu \mathrm{L}$ of whole blood according to the manufacturer's protocol using the QIA-amp DNA Mini Kit (QIAGEN, Hilden, Germany). Extracted DNA was quantified using a spectrophotometer at 260 and 280 NM with A260/A280 ratios between 1.6 and 1.8. Isolated DNA was diluted with $1 \mathrm{x}$ PCR buffer to $5 \mathrm{ng} / \mu \mathrm{L}$ and stored at $-20^{\circ} \mathrm{C}$ until analysis.

\section{High-resolution DNA-melting analysis}

Five primer pairs (A, B, C, D and E) (Table 1) were simultaneously used in PCRHRM to detect the beta thalassemia mutation commonly found in Thai populations.
The A-primer pairs were used for detection of a mutation in the HBB gene promoter region, e.g., -28A-G, $-31 \mathrm{~A}-\mathrm{G}$ and $-87 \mathrm{C}-\mathrm{A}$. The B-primer pairs were used for mutations in exon-1 and intron-1, e.g., CD17A-T, CD19A-G, CD26G-A, CD27/28+C, CD30G-A, IVS1-1G-T and IVS1-5G-C. The C-1 primer pairs were used for mutation in exon-2, namely, CD41/42-CTTT and CD71/72+A. The C-2 primer pairs were used for CD35C-A and CD41-C. The Dprimer pairs were used for mutation in intron-2, namely, IVS2-654C-T, and the Eprimer pairs were used for $3.4 \mathrm{~KB}$ deletion. The PCR-HRM test was initiated with the B and C-primer pairs. Each test protocol included one normal control and another unknown sample. The $25 \mu \mathrm{L}$ PCR-mixture for the PCR-HRM analysis included $5 \mu \mathrm{L}$ of DNA, $1.5 \mu \mathrm{M} \mathrm{MgCl} 2,200 \mu \mathrm{M}$ DNTP, 2 $\mu \mathrm{M}$ of SYTO9, 1 unit of Platinum Taq DNA polymerase, and $0.2 \mu \mathrm{M}$ of each primer, and the remaining volume was $1 x$ PCR buffer. Thermal cycling was performed using a Bio-Rad CFX96 Real-time System (BioRad Laboratories, 1000 Alfred Nobel Drive, Hercules, California 94547 USA) starting with an initial step of activating the Taq polymerase at $94^{\circ} \mathrm{C}$ for 2 minutes followed by 40 cycles of denaturation at $94^{\circ} \mathrm{C}$ for 15 seconds, annealing at $64^{\circ} \mathrm{C}$ for 15 seconds and extension at $72^{\circ} \mathrm{C}$ for 20 seconds. Fluorescence activity was measured using a SYBR Green1 channel $(533 \mathrm{~nm})$ at the end of each cycle. HRM analysis was performed according to the Bio-Rad Precision Melt Analysis Software (Bio-Rad Laboratories,). The image of the temperature-shift different curve developed was specific for each type of beta thalassemia mutation. ${ }^{15,16}$

\section{Direct DNA sequencing}

Two primer pairs were designed for the

Table 1. Primer sequences for PCR-HRM detection of common beta thalassemia mutations in Thailand.

\begin{tabular}{|c|c|c|c|}
\hline Primers & Nucleotide sequence $\left(5^{\prime}->3^{\prime}\right)$ & PCR size (bp.) & NCBI GenBank (U01317.1) \\
\hline A- forward & ACTTAGACCTCACCCTGTGGA & & $62022-42$ \\
\hline A-reverse & TGGTGTCTGTTTGAGGTTGC & 166 & $62167-87$ \\
\hline B -forward & CCTGAGGAGAAGTCTGCCGTT & & $62203-23$ \\
\hline B-reverse & GTCTCCACATGCCCAGTTTCT & 136 & $62320-41$ \\
\hline Cl-forward & CTGCTGGTGGT TAC CCTTG & & $62410-29$ \\
\hline Cl-reverse & AAAGGTGCCCTTGAGGTTGT & 166 & $62554-74$ \\
\hline C2-forward & TTCCCACCCTTAGGCTGCTGGT & & $62396-17$ \\
\hline C2-reverse & TGGCAAAGGTGCCCTTGAGGT & 183 & $62558-78$ \\
\hline D-forward & CTTTCTTTCAGGGCAATAATGA & & $63200-21$ \\
\hline D-reverse & AGAAATATTTATAGTCAGAAATATTG & 117 & $63290-316$ \\
\hline E1-forward & GTCACACTTTGGGTTGTAAGTGAC & & $61360-83$ \\
\hline E1-reverse & TCAATGTGCTCTGTGCATTAGT & 124 & $61461-83$ \\
\hline E2-forward & GTCACACTTTGGGTTGTAAGTGAC & & $61360-83$ \\
\hline E2-reverse & TTCCTTTTGTTGCCTTTGCT & 135 & $64960-79$ \\
\hline
\end{tabular}


molecular characterization of a beta thalassemia trait and/or disease. The S1-primer was for amplification of the HBB gene (NCBI GenBank U01317.1: forward primer nucleotide number 61974-93, reverse primer; 62700-20), which produced PCR products that were 780 base pairs in length. This segment was used for the detection of a beta thalassemia mutation in the promoter region, exon-1, intron-1 and exon-2 DNA segments. The S2-primer was used for amplification of the HBB gene (forward primer nucleotide number 663223-46, reverse primer; 63884-903), and the products were 660 base pairs in length. This segment was used for identification of a beta thalassemia mutation in the third part of the IVS2 nucleotide, exon-3 and poly-A tail region. The direct DNA sequencing process was performed according to the user's guide for the Applied Biosystems 3500 Genetic Analyzer. ${ }^{17}$

\section{Results}

Hemoglobin $\mathrm{A}_{2}$ proved beta thalassemia trait in 132 cases, hemoglobin E traits were identified in 338 cases, and 22 homozygous hemoglobin E cases were detected in 1,115 couples. PCR-HRM assessment revealed 12 types of beta thalassemia mutations, including hemoglobin $\mathrm{E}$, such as CD17 (A-T) 46 cases (35\%), CD41-42 (-CTTT), 45 cases (34\%), IVS-1 (G-T), 13 cases $(10 \%),-28$ (A-G) 12 , cases $(9 \%)$, CD35 (C-A), 4 cases (3\%), $3.4 \mathrm{~KB}$ deletion 4 (3\%) cases, CD 43 (G-T) 3 cases $(2 \%)$ and one case each $(1 \%)$ for CD 27-28 (+C), CD 71-72 (+A), IVS15 (G-C) and -87 (C-A) (Figures 1 and 2). The prevalence of beta thalassemia traits calculated was $5.9 \%$, and the prevalence of hemoglobin E trait was $17.1 \%$. A total of 23 at-risk couples were identified. Two couples were at-risk of having a baby with beta tha- lassemia major, one couple was at-risk of having a baby with beta thalassemia major or beta ${ }^{0}$-thalassemia/hemoglobin $\mathrm{E}$ disease, and the remaining couples were at risk of beta ${ }^{0}$-thalassemia/hemoglobin $\mathrm{E}$ disease. All subjects were on time for amniocentesis. Molecular analysis of isolated DNA from amniotic cells was performed using the HRM technique and direct DNA sequencing, which revealed that one fetus was homozygous CD17 (A-T), and 5 fetuses were affected by beta ${ }^{0}$ thalassemia/hemoglobin $\mathrm{E}$ disease. The beta ${ }^{0}$-thalassemia mutations in two cases of beta $^{0}$-thalassemia/hemoglobin $\mathrm{E}$ disease was CD41-42 (-CTTT), and one case each was CD17 (A-T), IVS1-(G-T) and CD35 (C-A). A one-year follow-up period for all babies born under the modified preventive strategy revealed no affected babies. The outcome of affected fetuses detected was in agreement with the calculations from the
A

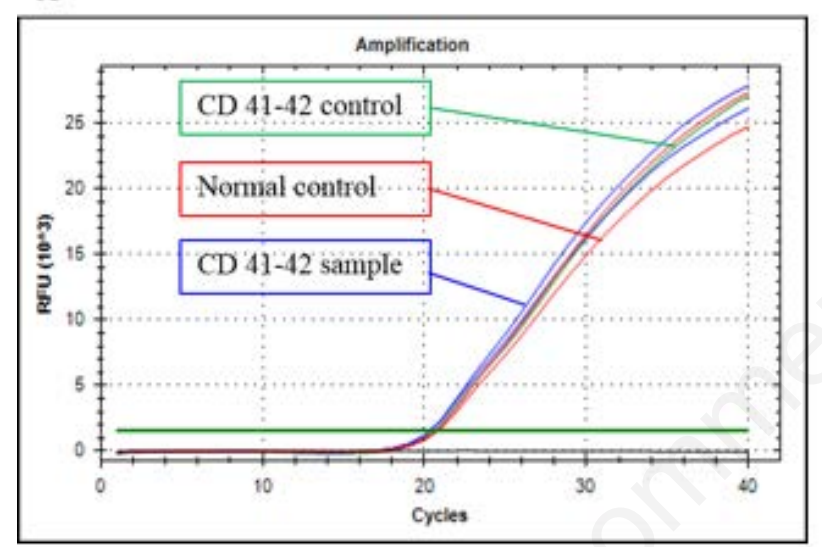

B

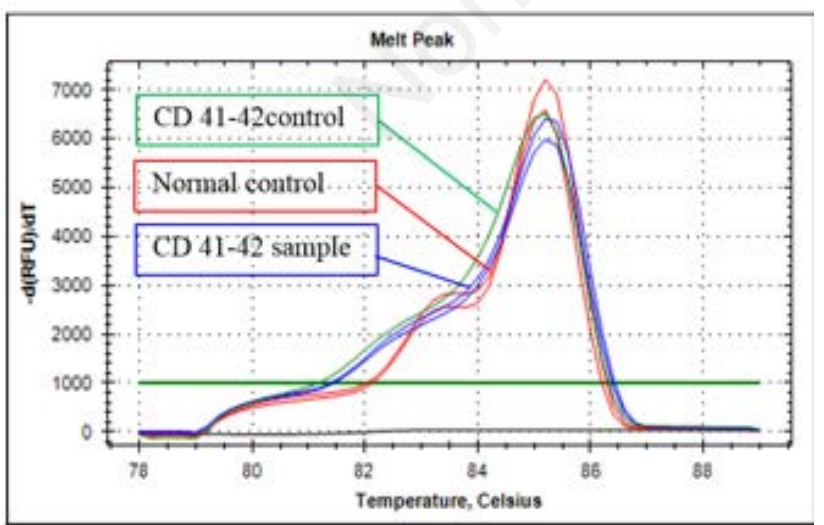

Figure 1. Quantitative real-time PCR image of a case of beta thalassemia trait CD41-42 -CTTT mutation tested using C1 primers. The red line represents normal control, green is the CD41-42-CTTT mutation, and blue is an CD 41-42 sample. Chromatogram $\mathrm{A}$ is an amplification profile, and $\mathrm{B}$ is a melting profile. In $\mathrm{B}$, it is not possible to differentiate the melting profiles between wild-type and mutant products.

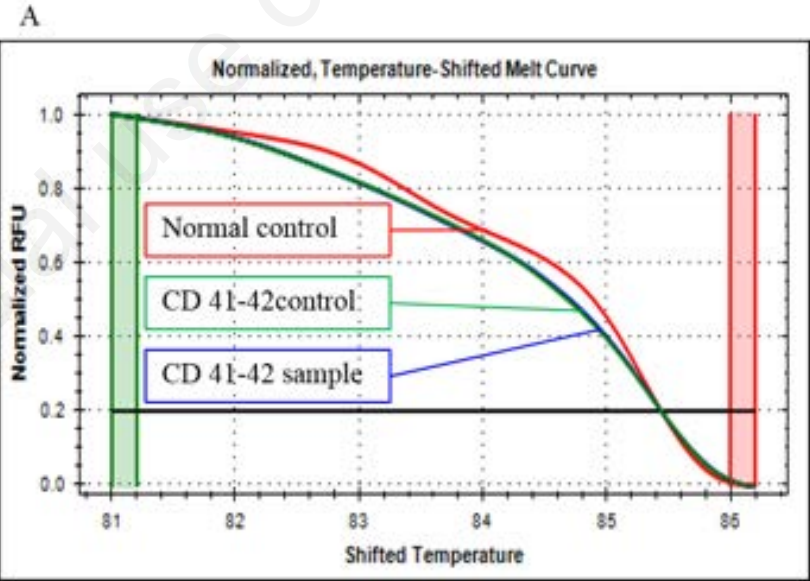

B

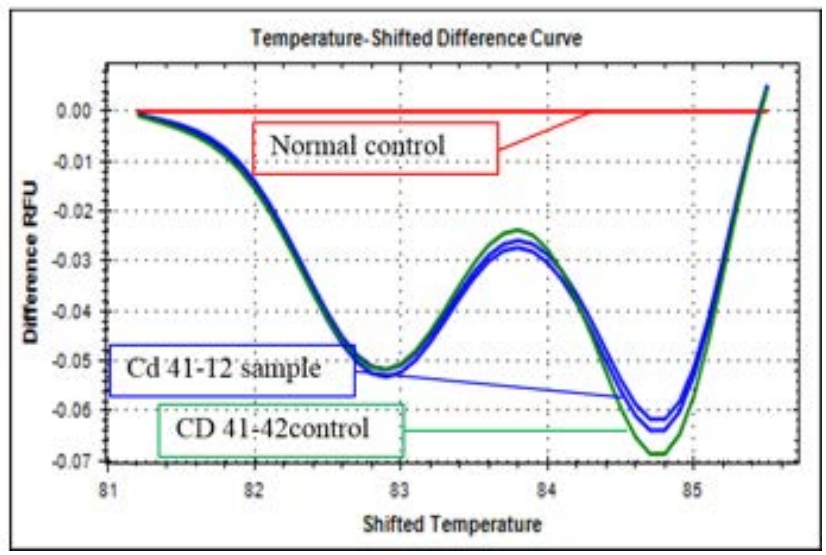

Figure 2. Chromatograms A and B are HRM analysis images that show a definite different pattern between wild-type and mutant products. Of the mutant products, the unknown melting profiles are in alignment with the genetically demonstrated CD 41-42 mutant, which suggests that the unknown mutant is a CD 41-42 mutation. 
Hardy-Weinberg equation for a recessive inherited single gene disorder.

\section{Discussion}

The most common thalassemia diseases in Southeast Asian countries are beta thalassemia major and beta thalassemia/hemoglobin E disease. Appropriate treatment for patients with these diseases is a substantial economic burden. ${ }^{18}$ Prevention is necessary, and it is good public health practice. The Thai national thalassemia prevention program was implemented in 2001. However, surveillance results in many Northern provinces of Thailand indicated that the thalassemia prevention program was not effective. Recently, in the year 2014-2015, the conventional screening policy for beta thalassemia prevention was performed in a provincial hospital, Phayao, Thailand. There were 1,067 pregnant couples recruited for at-risk fetuses with beta thalassemia disease. The laboratory methods involved were electronically determined RBC indices, single-tube osmotic fragility tests, single-tube DCIP tests and LPLC analysis of hemoglobin. Sixteen atrisk couples of beta thalassemia were detected. One fetus was affected by beta ${ }^{0}$ thalassemia/hemoglobin E disease after amniocentesis (Techa-aungkul 2017, personal communication). Analysis of the clinical records at the end of the prevention program revealed many reasons for screening failures. The major factors may be related to the complexity of the program activity, the responsibilities of the diagnostic laboratories and the thalassemia knowledge of the counselors. The minor factors included coinheritance of beta thalassemia trait with homozygous $\alpha^{+}$-thalassemia $(-\alpha /-\alpha)$ or alpha ${ }^{0}$-thalassemia trait $(--\alpha)$, which have significant effects on the red cell indices, particularly the MCV and $\mathrm{MCH}$, which may be normalized. The beta thalassemia trait may escape further evaluation in these cases. ${ }^{19}$ However, these factors must be defined to improve the effectiveness of the prevention programs.

A hemoglobin $\mathrm{A}_{2}$ level of $3.5 \%$ to $8 \%$ was an important turning point for the diagnosis of beta thalassemia trait. Therefore, all blood samples were assessed with the simultaneous use of Q-Sepharose microcolumn chromatography and capillary electrophoresis testing. Q-Sepharose micro-column chromatography was feasible for the screening of beta thalassemia traits and hemoglobin E carriers, and capillary electrophoresis testing was used for hemoglobin $\mathrm{A}_{2}$ confirmation. Capillary electrophoresis testing was very useful for the detection of abnormal hemoglobin and/or alpha thalassemia disease, which may modify hemoglobin $\mathrm{A}_{2}$ levels and also interfere the diagnosis of the beta thalassemia trait. ${ }^{20,21}$ There are two types of beta thalassemia trait, beta ${ }^{+}$ and beta ${ }^{0}$-thalassemia. The interaction of a beta $^{+}$-thalassemia mutation with beta ${ }^{0}$-thalassemia or hemoglobin E could ameliorate the clinical severity of beta thalassemia disease. Therefore, it is necessary to evaluate beta thalassemia mutations in all at-risk couples to provide appropriate thalassemia counseling. Couples with beta ${ }^{+}$-thalassemia mutation and the hemoglobin E carrier may not require prenatal diagnosis.

The PCR-HRM techniques using five primers (A, B, C, D and E) were designed across the beta HBB genes. At least 15 common beta thalassemia mutations were assessed. The HRM tests are a simpler and faster molecular technique that are suitable for identifying at-risk couples. The results of hemoglobin $\mathrm{A}_{2}$ quantification followed by PCR-HRM for molecular mutation assessment may be obtained within 7 days after receipt of blood samples. The fast reporting of a specific type of beta thalassemia mutation is very beneficial for thalassemia counseling. Therefore, the modified strategy enhanced the need of all pregnant women for early risk detection and early prenatal diagnosis.

\section{Conclusions}

Testing antenatal blood samples using HRM analysis after hemoglobin $\mathrm{A}_{2}$ estimation is a feasible laboratory method for the recruitment of couples at risk of having a fetus with beta thalassemia. The results were fast and accurate and may be obtained within 7 working days after receiving the sample. This modified strategy may be beneficial and improve the effectiveness of the beta thalassemia prevention program.

\section{References}

1. Fucharoen S, Winichagoon P. Haemoglobinopathies in SoutheastAsia. Indian J Med Res 2011;134:498506.

2. Boonsa S, Sanchaisuriya K, Fucharoen $\mathrm{G}$, et al. The diverse molecular basis and hematological features of $\mathrm{Hb} \mathrm{H}$ and AEBart's diseases in Northeast Thailand. Acta Haematol 2004;111:149-54.

3. Charoenkwan P, Taweephon R, SaeTung R, et al. Molecular and clinical features of $\mathrm{Hb} \mathrm{H}$ disease in northern Thailand. Hemoglobin 2005;29:133-40

4. Fucharoen S, Viprakasit V. Hb H disease: clinical course and disease modifiers. Hematology Am Soc Hematol Educ Program 2009:26-34.

5. Galanello R, Origa R. Beta-thalassemia. Orphanet J Rare Dis 2010;5:11.

6. Weatherall DJ. Thalassemia as a global health problem: recent progress toward its control in the developing countries. Ann N Y Acad Sci 2010;1202:17-23.

7. Charoenkwan P, Teerachaimahit P, Sanguansermsri T. The correlation of $\alpha$ globin gene mutations and the XmnI polymorphism with clinical severity of $\mathrm{Hb}$ E/ $\beta$-thalassemia. Hemoglobin 2014;38:335-8.

8. Fucharoen S, Weatherall DJ. Progress toward the control and management of the thalassemias. Hematol Oncol Clin North Am 2016;30:359-71.

9. Tienthavorn V, Pattanapongsthorn J, Charoensak S, et al. Prevalence of Thalassemia Carriers in Thailand. Thai J Hematol Transf Med 2006;16:307-12.

10. Tongsong T, Wanapirak C, Sirivatanapa $\mathrm{P}$, et al. Prenatal control of severe thalassaemia: Chiang Mai strategy. Prenat Diagn 2000;20:229-34.

11. Wong $P$, Sritippayawan S, Suwannakhon N, et al. Q-Sepharose micro-column chromatography: a simple screening method for identifying beta thalassemia traits and hemoglobin E carriers. Clin Biochem 2016;49:128891.

12. Hafiza A, Malisa MY, Khirotdin RD, et al. HbA2 levels in normal, beta-thalassaemia and haemoglobin E carriers by capillary electrophoresis. Malays J Pathol 2012;34:161-4.

13. Sangkitporn S, Sangkitporn SK, Tanjatham S, et al. Multicenter validation of fully automated capillary electrophoresis method for diagnosis of thalassemias and hemoglobinopathies in Thailand. Southeast Asian J Trop Med Public Health 2011;42:1224-32.

14. Mais DD, Gulbranson RD, Keren DF. The range of hemoglobin A2 in hemoglobin $\mathrm{E}$ heterozygotes as determined by capillary electrophoresis. Am J Clin Pathol 2009;32:34-8.

15. Charoenkwan P, Sirichotiyakul S, Phusua A, et al. High-resolution melting analysis for prenatal diagnosis of betathalassemia in northern Thailand. Int $\mathrm{J}$ Hematol 2017;106:757-64.

16. Saetung R, Ongchai S, Charoenkwan P, Sanguansermsri T. Genotyping of beta thalassemia trait by high-resolution DNA melting analysis. Southeast Asian J Trop Med Public Health 
2013;44:1055-64.

17. Sirichotiyakul S, Saetung R, Sanguansermsri T. Analysis of beta-thalassemia mutations in northern Thailand using an automated fluorescence DNA sequencing technique. Hemoglobin 2003;27:89-95.

18. Riewpaiboon A, Nuchprayoon I, Torcharus K, et al. Economic burden of beta-thalassemia/Hb $\mathrm{E}$ and beta-tha- lassemia major in Thai children. BMC Res Notes 2010;3:29.

19. Viprakasit V, Limwongse C, Sukpanichnant $\mathrm{S}$, et al. Problems in determining thalassemia carrier status in a program for prevention and control of severe thalassemia syndromes: a lesson from Thailand. Clin Chem Lab Med 2013;51:1605-14.

20. Singha K, Srivorakun H, Fucharoen G,
Fucharoen S. Co-inheritance of $\alpha 0$-thalassemia elevates $\mathrm{Hb} \mathrm{A} 2$ level in homozygous $\mathrm{Hb} \mathrm{E}$ : Diagnostic implications. Int J Lab Hematol 2017;39:50812.

21. Panyasai S, Sukunthamala K, Jaiping $\mathrm{K}$, et al. Interference of hemoglobin Hope on beta-thalassemia diagnosis by the capillary electrophoresis Method. Am J Clin Pathol 2011;136:14-8. 